\title{
Análisis de la bondad de múltiples indicadores para el diagnóstico de la adicción al cibersexo*
}

\author{
Jesús Castro-Calvo ${ }^{1}$ (jesus.castro@uv.es), Marta García-Barba ${ }^{2}$, Verónica Cervigón \\ Carrasco $^{2}$ y Rafael Ballester-Arnal ${ }^{2}$
}

\footnotetext{
${ }^{1}$ Salusex, Dpto. Personalidad, Evaluación y Tratamientos Psicológicos, Universitat de València.

${ }^{2}$ Salusex, Dpto. Psicología Básica, Clínica y Psicobiología. Universitat Jaume I
}

\section{Resumen}

El uso de Internet con propósitos sexuales (p.e., visionado de pornografía o contacto sexual mediante chat o cámara web) resulta una práctica cada vez más generalizada. Si bien en la mayoría de los casos se realiza de forma recreativa, en algunas ocasiones puede sobrevenir compulsivo, incontrolado y asociado a deterioro funcional en varios ámbitos (adicción al cibersexo). Las escalas de las que disponemos para su diagnóstico se han visto limitadas a la hora de identificar estos pacientes debido a que algunos indicadores diagnósticos han perdido especificidad: por ejemplo, el tiempo online con fines sexuales parece no discriminar usuarios problemáticos debido al uso cada vez más intensivo de cibersexo. El objetivo de este trabajo es analizar la bondad de diferentes indicadores diagnósticos a la hora de identificar perfiles de consumo adictivo mediante análisis clúster. A tal fin, 1000 hombres (18-35 años) completaron una batería de cuestionarios que evaluaba múltiples indicadores de consumo. El análisis clúster reveló la existencia de 2 perfiles: usuarios recreativos (51.8\%) y posiblemente problemáticos (48.2\%). Ambos perfiles únicamente diferían entre sí en cuanto a percepción de consumo problemático, niveles globales de hipersexualidad, puntuación en una escala de adicción al cibersexo y percepción de interferencia (ordenados en función de su relevancia diagnóstica). El hecho de que únicamente emerjan dos perfiles de consumo que además difieren poco entre ellos sugiere que cada vez es más difícil distinguir el consumo problemático del recreativo.

Palabras clave: Cibersexo; actividad sexual online; adicción al cibersexo; diagnóstico.

*Este trabajo se ha realizado gracias a dos proyectos de investigación financiados por la Universitat Jaume I de Castellón (P1.1B2012-49 y P1.1B2015-82). 
Title: Goodness-of-fit analysis of multiple indicators for the diagnosis of cybersex addiction.

\begin{abstract}
The use of the Internet for sexual purposes (e.g., viewing pornography or sexual contact via chat or webcam) is an increasingly widespread practice. Even though in most cases this is recreational, in some cases may become compulsive, uncontrolled and associated with functional impairment in several areas (cybersex addiction). The scales for diagnosis have been limited in identifying these patients because some diagnostic indicators have lost specificity: for example, online time for sexual purposes does not seem to discriminate problematic users due to the increasingly intensive use of cybersex. The aim of this work is to analyze the goodness of fit of different diagnostic indicators in identifying addictive consumption profiles by means of cluster analysis. For this purpose, 1000 men (18-35 years old) completed a battery of questionnaires assessing multiple indicators of consumption. The cluster analysis revealed the existence of 2 profiles: recreational users (51.8\%) and possibly problematic users $(48.2 \%)$. Both profiles only differed from each other in terms of perceived problematic use, overall levels of hypersexuality, score on a cybersex addiction scale, and perceived interference (ranked in order of diagnostic relevance). The fact that only two consumption profiles emerge that differ little from each other suggests that it is increasingly difficult to distinguish problematic from recreational use.
\end{abstract}

Keywords: cybersex, online sexual activities, cybersex addiction, diagnosis. 


\section{Introducción}

Las tecnologías de la Información y la Comunicación (TICs) abarcan cada día áreas más amplias de nuestra cotidianeidad, y las relaciones afectivo-sexuales -en general-y el sexo -en concreto- no han quedado al margen de este proceso de "colonización digital". Cada vez son más las personas que usan Internet para obtener información sobre sexo y sobre sexualidad, "preparándose" de este modo para sus primeros encuentros sexuales; también es cada vez más frecuente que Internet sea el lugar en el que conocer a potenciales parejas, así como el medio donde interactuar con ellas y llevar a cabo el cortejo (sería, por ejemplo, el caso de las populares apps para ligar); finalmente, en Internet existen también múltiples formas de obtener la gratificación sexual, tanto en solitario como con otros usuarios. Así, las TIC abarcarían hoy en día prácticamente todos los ámbitos de la sexualidad.

En este contexto de creciente "digitalización" de la sexualidad, el equipo de investigación SALUSEX (Universitat Jaume I y Universitat de València) lleva casi dos décadas analizando, a través de investigaciones empíricas en muy diversas poblaciones y con múltiples objetivos, el uso del sexo online de los españoles. Estas investigaciones han permitido ampliar nuestro conocimiento en torno a temas tan relevantes como cuál es la prevalencia de usuarios recreativos de cibersexo y consumidores problemáticos (es decir, personas que muestran un consumo abusivo o adictivo del sexo online), qué aspectos es conveniente tener en cuenta a la hora de discriminar entre estos perfiles y cómo podríamos ayudar a aquellas personas en las que se confirma la presencia de consumo problemático. En este artículo, se abordarán estos aspectos a través de la presentación de los principales hallazgos realizados por el equipo SALUSEX en sus diversos estudios. Asimismo, se presentan también datos de un estudio nacional para hablar de un aspecto más concreto: en qué indicadores diagnósticos deberíamos fijarnos para identificar si una persona presenta un perfil de consumo problemático de cibersexo. A propósito de estos datos, finalmente se esbozarán algunas conclusiones acerca de la evaluación y tratamiento de la adicción al cibersexo.

\section{Conceptualización, prevalencia y hábitos de consumo de sexo online en España}

Durante los últimos años, se ha popularizado el uso del término cibersexo para designar la intersección entre las TIC y la sexualidad (Ballester-Arnal, Castro-Calvo, Gil-Llario, et al., 2020). Desde una perspectiva amplia, el cibersexo comprendería cualquier tipo de 
actividad que suponga el «uso de Internet con objetivos de gratificación sexual» (Cooper \& Griffin-Shelley 2002). En esta definición caben gran variedad de Actividades Sexuales Online (ASO), a las que se irían sumando otras a medida que el desarrollo de la tecnología facilita nuevas formas de sexualidad online. A fin de organizar esta enorme diversidad de conductas sexuales que tienen lugar en Internet, se han propuesto varias taxonomías que clasifican las ASO en función de dos criterios: si se llevan a cabo con el fin de obtener la excitación y gratificación sexual y si su realización implica interacción con otros usuarios (Shaughnessy et al., 2011). El cruce entre estos criterios permitiría establecer tres grandes categorías de ASO: (1) ASO cuyo fin último no es lograr el placer sexual y la excitación, (2) ASO que buscan el placer y la excitación mediante actividades sexuales en solitario (o "cibersexo solitario") y (3) ASO que también persiguen este objetivo, pero a través de actividades sexuales con otros usuarios ("cibersexo social").

La primera de estas categorías permite abordar un aspecto importante: no todo lo que las personas hacen online relacionado con la sexualidad se hace con finalidad hedónica -esto es, de búsqueda inmediata del placer-. Una de las ASO más comunes englobadas en esta categoría sería la búsqueda de información sobre sexo y sexualidad: cada vez son más los jóvenes que por anonimato, comodidad o por cubrir las deficiencias de nuestros sistemas educativos a la hora de dotar de educación afectivo-sexual, optan por usar Internet como forma de aprender sobre sexo y sexualidad (Simon et al., 2015). En la categoría de ASO

que no requieren contacto con otros usuarios -cibersexo solitario-, destacaría la descarga y el visionado de pornografía (presumiblemente por la facilidad para acceder anónima y gratuitamente a este tipo de contenidos). La realización de esta ASO se suele acompañar de masturbación, de modo que la persona que la realiza suele obtener gratificación sexual inmediata asociada a este comportamiento. Finalmente, entre las que implican interacción con otros usuarios (o cibersexo social), deberíamos distinguir entre aquellas que también se suelen acompañar de masturbación (como mantener conversaciones sexuales con otros usuarios -mediante chat o webcam-o intercambiar imágenes sexuales a través del móvil -Sexting-) y las que no. Entre estas últimas, destacaría por ejemplo el uso de aplicaciones móviles basadas en geolocalización (p.e., Tinder o Grindr) para conocer personas con las que iniciar una relación romántica o sexual. En este último caso, la gratificación sexual no se produce durante la realización 
la ASO, sino a posteriori (cuando el usuario contacta offline con la persona que ha conocido).

Una de las líneas de investigación más activas del equipo SALUSEX ha sido precisamente la de cuantificar la prevalencia de estas ASO. En este sentido, contamos con datos de tres estudios distintos que nos han permitido: (1) analizar las principales características del consumo de cibersexo en jóvenes con y sin pareja estable (Ballester-Arnal et al., 2014); (2) estimar la prevalencia de distintas ASO y del uso problemático en función del género (Ballester-Arnal, Castro-Calvo, et al., 2016); (3) estimar estas mismas prevalencias pero en población adolescente (Ballester-Arnal, Giménez-García, et al., 2016); (4) comparar la prevalencia a lo largo del ciclo vital (Ballester-Arnal et al., 2021); y (5) contrastar si el consumo de cibersexo en nuestro país ha cambiado durante la última década (Ballester-Arnal, Castro-Calvo, Gil-Llario, et al., 2020). Estos tres estudios, realizados en tres franjas temporales distintas (entre 2009 y 2010, entre 2012 y 2013 y entre 2016-2021), sumarían una muestra de aproximadamente 12,000 participantes. Las dos investigaciones que ofrecen las conclusiones más relevantes serían las del estudio en el que comparamos el consumo de cibersexo a lo largo del ciclo vital en una muestra representativa de en torno a 8,000 españoles (Ballester-Arnal et al., 2021) y el estudio en el que comparamos el consumo de cibersexo en España en 2009 y 2019 (Ballester-Arnal, Castro-Calvo, Gil-Llario, et al., 2020). A continuación, resumimos sus conclusiones:

- La prevalencia de consumo de pornografía alcanzaría el $96 \%$ en hombres y el $73 \%$ en mujeres (es decir, que prácticamente toda la población masculina y una buena proporción de la femenina consume pornografía).

- Le seguirían en prevalencia la búsqueda de información sobre sexo y sexualidad y el uso de chats o webcam para interactuar con fines sexuales.

- La franja de edad en la que se consume una mayor cantidad de cibersexo es entre los 18 y los 40 años, si bien las diferencias en función de la edad son pequeñas (es decir, que el consumo de cibersexo se mantiene con apenas variaciones desde la adolescencia hasta prácticamente la vejez).

- Si bien la brecha entre hombres y mujeres se mantiene, parece que las diferencias se van atenuando debido al aumento de la prevalencia de ASO entre estas últimas. 
- Durante la última década, la prevalencia de consumo de pornografía aumentó un $49,2 \%$ en hombres y un $58,9 \%$ en mujeres.

- De forma similar, la participación en chats sexuales creció en esta década un $22,2 \%$ en hombres y $28,6 \%$ en mujeres.

\section{Perfiles de consumo de cibersexo}

Existe un cierto debate en torno a los beneficios y los inconvenientes del sexo en Internet, sobre todo si hablamos del impacto en el desarrollo psicosexual de jóvenes y adolescentes (O’Sullivan, 2014). Entre sus beneficios, algunos investigadores sostienen que el sexo en Internet constituye un medio eficaz para la satisfacción del deseo sexual (Daneback et al., 2013), compensar la escasez de conocimientos sobre sexualidad (Smith, 2013), encontrar parejas románticas o sexuales en un entorno seguro (Courtice \& Shaughnessy, 2018) o para distraerse del aburrimiento y de los problemas cotidianos (Castro-Calvo et al., 2018).

En el extremo contrario, el sexo en Internet también puede llegar a provocar problemas a sus usuarios en función de dos aspectos: el tipo de actividades sexuales online realizadas o la manera en la que éstas se realizan. Así, ciertas ASO realizadas en colectivos concretos (como el sexting en adolescentes) se consideran problemáticas per se independientemente de que se realicen esporádicamente o con más frecuencia (Cooper et al., 2016). Asimismo, ASO mayoritarias (como el consumo de pornografía) pueden sobrevenir un problema cuando se realizan de forma abusiva; así, uno de los principales riesgos del sexo online es precisamente la posibilidad de desarrollar un cuadro clínico de adicción al cibersexo. La adicción al cibersexo se definiría como el "uso excesivo e incontrolado de cibersexo que provoca problemas laborales, sociales y/o personales" (Cooper \& Griffin-Shelley, 2002). Según Cooper (1998), los pacientes con adicción al cibersexo pasarían una gran cantidad de tiempo realizándolo, persisten a pesar de sus consecuencias negativas, no tienen control sobre su inicio o finalización, negarían su problema y fallarían en el intento por controlar su consumo. En la mayoría de los casos, el cuadro clínico no aparece de forma aguda, sino que seguiría una progresión insidiosa: comienza con un consumo recreativo, se exacerba en frecuencia y severidad con el tiempo hasta acabar finalmente resultando adictivo (Reid \& Kafka, 2014). Eso permite identificar los tres perfiles clásicos de consumo de cibersexo: usuarios recreativos (es decir, personas que lo consumen sin que suponga problema 
alguno), de riesgo (personas que comienzan a consumirlo de forma más persistente y a experimentar los primeros problemas asociados a su consumo pero con una gravedad moderada y manteniendo un cierto control) y adictos al cibersexo (pacientes en los que el cuadro clínico se manifiesta con suficiente gravedad como para su diagnóstico) (Ballester-Arnal, Castro-Calvo, Gil-Llario, et al., 2020).

Desde SALUSEX, otra de las líneas de investigación prioritarias ha sido el análisis de la proporción de usuarios de cibersexo que se podrían clasificar en cada uno de estos perfiles. A nivel internacional, se estima que la prevalencia de este cuadro clínico se sitúa entre el 1.7\%-7.6\% (Ross et al., 2012). Si bien sus características son cualitativa y cuantitativamente diferentes, dentro de este porcentaje se englobaría tanto a los usuarios de riesgo como a los patológicos. En nuestros estudios, hemos tratado de distinguir entre ellos, ajustando nuestras estadísticas al tipo de perfil. En adolescentes, la prevalencia de usuarios de riesgo se situaba en el $8.6 \%$ en chicos y del $2.2 \%$ en chicas (Ballester-Arnal, Giménez-García, et al., 2016). En adultos, lo que encontramos fue que el 90\% mostró un perfil de bajo riesgo de adicción al cibersexo, el 8.6\% de riesgo medio (usuarios de riesgo) y el $0.7 \%$ superó el corte para su clasificación como adictos al cibersexo (Ballester-Arnal, Castro-Calvo, et al., 2016).

\section{Adicción al cibersexo: validez y eficiencia de diferentes indicadores diagnósticos}

Otra de las líneas de investigación en las que el equipo SALUSEX trabaja más activamente es la de la identificación de aspectos del comportamiento sexual online que puedan constituir buenos indicadores -es decir, indicadores sensibles y específicos-de consumo problemático de cibersexo. Evidentemente, los indicadores más precisos a la hora de identificar a usuarios problemáticos de cibersexo (especialmente, a aquellos cuya sintomatología sería de tal gravedad como para diagnosticar un cuadro clínico de adicción al cibersexo) serían los propios signos/síntomas de esta condición. En este sentido, los síntomas centrales son: a) la persona dedica un excesivo tiempo y/o esfuerzo al consumo de cibersexo, b) experimenta problemas para controlarlo (p.e., para decidir su inicio/finalización), c) tiene dificultad para atender obligaciones familiares, académicas o laborales y d) persiste en la conducta sexual a pesar de las consecuencias. A eso se suma un quinto criterio, que sería el uso del cibersexo como regulador emocional. 
Además de los signos y síntomas mencionados, durante largo tiempo se consideró que la frecuencia de ASO constituía un criterio robusto para identificar a los adictos al cibersexo, argumentando que solo una persona que invierta una gran cantidad de tiempo online con fines sexuales podía ser candidata a este diagnóstico. El principal valedor de esta postura era Cooper, que en sus investigaciones proponía que dedicar más de 11 horas semanales al consumo de cibersexo era el indicador más sólido de una adicción (Cooper et al., 2000). Esta postura recibió cierto respaldo de investigaciones posteriores. Así, Voon et al. (2014) analizaron el tiempo semanal dedicado a la ASO de los pacientes con y sin problemas en el control de los impulsos sexuales, encontrando que los pacientes clínicos invertían una media de 13.2 horas semanales (frente a 1.7 horas semanales en población normal). En nuestros estudios con muestra española, la correlación entre tiempo invertido online con fines sexuales y gravedad del consumo (medida a partir del Internet Sex Screening Test, ISST) era de .45, lo que significa que la varianza compartida entre ambas variables era de cerca del 20\% (Ballester-Arnal, Castro-Calvo, et al., 2016). Desde la otra perspectiva, este dato indicaría que cerca del $80 \%$ de varianza del consumo problemático de cibersexo vendría explicada por factores ajenos al tiempo invertido online. Esta baja validez diagnóstica puede deberse al cambio en los patrones de consumo de cibersexo producidos durante la última década: dado que cada vez más gente consume cibersexo (y además lo hace invirtiendo cantidades de tiempo cada vez mayores), este aspecto podría haber dejado de discriminar adecuadamente entre usuarios recreativos y problemáticos. Esta fue la conclusión a la que llegaron Bőthe et al. (2020), que tras aplicar análisis de clúster en una muestra de más de 15,000 participantes, concluyeron que el tiempo online ya no permitía discriminar entre usuarios recreativos y problemáticos.

Otros estudios sugieren que la relación entre tiempo online con fines sexuales y consumo problemático se explica a través de dos vías complementarias: una directa y otra indirecta. En cuanto a la vía directa, pasar más tiempo realizando la ASO se relaciona positivamente -aunque de forma moderada- con la pérdida del control sobre el impulso sexual $(r=.30)$. A nivel indirecto, pasar más cantidad de tiempo consumiendo cibersexo aumentaría la percepción subjetiva de adicción; a su vez, esto se relacionaría con una pérdida real del control de impulsos sexuales y con el empeoramiento de la satisfacción sexual (Blais-Lecours et al., 2016), de modo que lo que podría estar 
determinando realmente la relación entre el tiempo y la gravedad del consumo no sería tanto la interferencia derivada de la dedicación temporal como el hecho de que una persona considere que padece un problema con su consumo de cibersexo. Este no es el único estudio que apostaría por la percepción subjetiva de consumo problemático como indicador de adicción (Grubbs et al., 2019). Sin embargo, apenas existen investigaciones que comparen su bondad diagnóstica con la de los criterios clásicos de adicción (signos y síntomas de adicción al cibersexo).

Por último, algunos han apostado por valerse de otros indicadores a la hora de discriminar entre usuarios recreativos y problemáticos de cibersexo. Como ya se ha mencionado, las personas con un patrón de consumo adictivo presentan un perfil psicosocial determinado: concretamente, estos usuarios son, en su mayoría, hombres, suelen presentar niveles altos de hipersexualidad y de búsqueda de sensaciones sexuales y peores niveles de autoestima (Castro-Calvo et al., 2020). Este tipo de indicadores por supuesto no cumplirían el criterio de especificidad del que hablábamos anteriormente (es decir, que también aparecerían en otros cuadros clínicos), pero es posible que considerarlos como indicios complementarios para cribar usuarios recreativos $\mathrm{y}$ problemáticos mejore la precisión de las formulaciones diagnósticas.

Existen múltiples procedimientos para identificar perfiles de riesgo y examinar la bondad diagnóstica de diferentes signos y síntomas. El análisis clúster es uno de los más populares ya que permite utilizar múltiples indicadores (tanto de tipo categórico como dimensional) para identificar subgrupos de personas que comparten una serie de características que los distinguen de otros subgrupos (Henry et al., 2005). De modo que este método permitiría hacer cribados masivos (es decir, en muestras de gran tamaño) para detectar la presencia de subgrupos (como sería el caso de los usuarios problemáticos o los adictos al cibersexo). Desde el equipo de investigación SALUSEX, hemos usado con éxito este método para identificar subgrupos de individuos con problemas en el control de impulsos sexuales (es decir, adictos al sexo) en muestras de gran tamaño (Ballester-Arnal, Castro-Calvo, Giménez-García, et al., 2020; Castro-Calvo et al., 2020). Sin embargo, hasta este momento, no lo habíamos utilizado para identificar a usuarios problemáticos de cibersexo y testar la eficacia comparada de varios indicadores diagnósticos (incluyendo indicadores relacionados con las características del consumo -por ejemplo, el tiempo semanal online dedicado al 
consumo de cibersexo-, indicadores de consumo psicopatológico -los signos y síntomas clásicos de adicción- y otras variables y disposiciones asociadas al consumo adictivo -como el nivel de hipersexualidad y autoestima-). A tal fin, seleccionamos a una submuestra de 1,000 hombres reclutados para nuestro estudio nacional sobre consumo de cibersexo (para una descripción completa del procedimiento y metodología del estudio, ver Ballester-Arnal et al., 2021). Todos ellos completaron una batería de escalas que nos permitieron obtener hasta 15 indicadores directos e indirectos de consumo problemático de cibersexo. Todos estos indicadores se analizaron mediante el método de clasificación clúster en dos pasos. El análisis de clúster reveló la existencia de dos perfiles únicamente: usuarios recreativos (51.8\%) y posiblemente problemáticos (48.2\%). El hecho de que el clúster de "posiblemente problemáticos" agrupe a casi el $50 \%$ de la muestra, junto con el pobre ajuste que mostraba la medida de la silueta de cohesión y separación (0.2), sugeriría que los indicadores utilizados (o, al menos, un gran número de ellos) no serían apropiados para distinguir entre usuarios recreativos de cibersexo y usuarios problemáticos o adictos. A continuación se presenta una figura con los resultados en cuanto a la calidad diagnóstica de cada uno de los 15 indicadores utilizados (figura 1): 
De este estudio se derivan algunas conclusiones interesantes. En primer lugar, parece que algunos indicadores clásicos de consumo problemático o adictivo de cibersexo (como el tiempo online con fines sexuales, el tipo de ASO realizada o las preferencias por diferente tipo de contenidos sexuales) resultarían prácticamente irrelevantes. Asimismo, el nivel de autoestima tampoco permitiría discriminar entre perfiles de consumo de cibersexo. Otra de las conclusiones principales de este estudio es que el nivel de hipersexualidad y, sobre todo, de signos y síntomas de consumo adictivo de cibersexo sólo tendrían una capacidad media de discriminación entre clústeres. Esto último es especialmente relevante, ya que vendría a sugerir que los instrumentos de evaluación de síntomas de los que nos valemos para el diagnóstico de esta condición podrían no ser eficaces para distinguir entre perfiles de consumo. En el extremo contrario, los mejores indicadores de consumo problemático fueron los relacionados con la autopercepción de consumo problemático (en concreto, la percepción de dedicación excesiva, de consumo adictivo y de interferencia).

\section{Conclusiones}

De lo expuesto hasta el momento, se derivarían algunas conclusiones que modulan cómo debería ser nuestro abordaje clínico del consumo de cibersexo. En primer lugar, el uso de Internet con fines sexuales es una práctica extremadamente extendida (como hemos visto, prácticamente todas las personas han realizado, a lo largo de su vida, algún tipo de ASO) (Ballester-Arnal et al., 2021). En la mayoría de los casos (en torno al 90\% de usuarios), el consumo de cibersexo es recreativo y no reporta problema alguno; sin embargo, el $10 \%$ restante tendría una alta probabilidad de desarrollar $-\mathrm{o}$ ya manifestaría- un cuadro clínico relacionado con el abuso del sexo online. De modo que, si bien la proporción de usuarios problemáticos no sería especialmente alta, el número bruto de personas susceptibles de recibir una evaluación e incluso un tratamiento por un posible problema de adicción sería considerable. En este contexto, identificar en qué perfil de consumo (recreativo, de riesgo o adicto) se encuadra cada usuario resulta esencial. El problema es que discriminar entre estos perfiles no sería tan sencillo como podría parecer a priori. Por ejemplo, los síntomas clásicos para diagnosticar la adicción al cibersexo o el consumo problemático, medidos a través de cuestionarios y escalas diagnósticas, paradójicamente parece que no serían los mejores indicadores para discriminar entre los distintos perfiles de consumo. Asimismo, otros indicadores comúnmente utilizados para el diagnóstico de la adicción al cibersexo (como el tiempo 
online con fines sexuales o aspectos relacionados con las características de consumo) muestran incluso peor capacidad de discriminación. En el extremo contrario, parece que los indicadores relacionados con la autopercepción de consumo problemático serían los mejor posicionados a la hora de discriminar entre perfiles. Así, en un contexto en el que cada vez resulta más complicado establecer los límites entre consumo recreativo y problemático, las evaluaciones deberían tener en cuenta muy diversos aspectos a la hora de juzgar en qué perfil encajaría el patrón de consumo de cibersexo de cada paciente. Esta es también la filosofía que ha guiado la elaboración por parte del equipo de investigación SALUSEX de uno de los programas más pioneros para el tratamiento de esta condición clínica (Ballester-Arnal, Castro-Calvo, Gil-Llario, et al., 2020). Este programa, basado en una aproximación terapéutica cognitivo-conductual, estructurado y administrable en 12 sesiones, aborda de forma integral todos estos aspectos a fin de ofrecer una propuesta que resulte eficaz y adaptada al perfil concreto de cada usuario de cibersexo.

\section{Referencias}

Ballester-Arnal, R., Castro-Calvo, J., García-Barba, M., Ruiz-Palomino, E., y Gil-Llario, M. D. (2021). Problematic and non-problematic engagement in Online Sexual Activities across the lifespan. Computers in Human Behavior, 120. https://doi.org/10.1016/j.chb.2021.106774

Ballester-Arnal, R., Castro-Calvo, J., Gil-Llario, M. D., y Gil-Juliá, B. (2016). Cybersex Addiction: A Study on Spanish College Students. Journal of Sex \& Marital Therapy, 43(6), 567-584. https://doi.org/10.1080/0092623X.2016.1208700

Ballester-Arnal, R., Castro-Calvo, J., Gil-Llario, M. D., y Giménez-García, C. (2014). Relationship status as an influence on cybersex activity: cybersex, youth, and steady partner. Journal of Sex \& Marital Therapy, 40(5), 444-456. https://doi.org/10.1080/0092623X.2013.772549

Ballester-Arnal, R., Castro-Calvo, J., Gil-Llario, M. D., y Giménez-García, C. (2020). Adicción al cibersexo: teoría, evaluación y tratamiento. Alianza Editorial.

Ballester-Arnal, R., Castro-Calvo, J., Giménez-García, C., Gil-Juliá, B., y Gil-Llario, M. D. (2020). Psychiatric comorbidity in Compulsive Sexual Behavior Disorder (CSBD). Addictive Behaviors, 107. https://doi.org/10.1016/j.addbeh.2020.106384

Ballester-Arnal, R., Giménez-García, C., Gil-Llario, M. D., y Castro-Calvo, J. (2016). Cybersex in the "Net generation": Online sexual activities among Spanish 
adolescents. Computers in Human Behavior, 57, 261-266. https://doi.org/10.1016/j.chb.2015.12.036

Blais-Lecours, S., Vaillancourt-Morel, M.-P., Sabourin, S., y Godbout, N. (2016). Cyberpornography: Time Use, Perceived Addiction, Sexual Functioning, and Sexual Satisfaction. Cyberpsychology, Behavior, and Social Networking, 19(11), 649-655. https://doi.org/10.1089/cyber.2016.0364

Bőthe, B., Tóth-Király, I., Potenza, M. N., Orosz, G., y Demetrovics, Z. (2020). High-Frequency Pornography Use May Not Always Be Problematic. The Journal of Sexual Medicine, 17(4), 793-811. https://doi.org/10.1016/j.jsxm.2020.01.007

Castro-Calvo, J., Gil-Llario, M. D., Giménez-García, C., Gil-Juliá, B., y Ballester-Arnal, R. (2020). Ocurrence and clinical characteristics of Compulsive Sexual Behavior Disorder (CSBD): a cluster analysis in two independent community samples. Journal of Behavioral Addictions, 9(12), 446-468. https://doi.org/10.1556/2006.2020.00025

Castro-Calvo, J., Giménez-García, C., Gil-Llario, M. D., y Ballester-Arnal, R. (2018). Motives to engage in Online Sexual Activities and their links to an excessive and problematic use: a Systematic Review. Current Addiction Reports, 5(4), 491-510. https://doi.org/https://doi.org/10.1007/s40429-018-0230-y

Cooper, A. (1998). Sexually Compulsive Behavior. Contemporary Sexuality, 32(4), 1-3. Cooper, A., Delmonico, D. L., y Burg, R. (2000). Cybersex users, abusers, and compulsives: New findings and implications. Sexual Addiction \& Compulsivity, 7(1-2), 5-29. https://doi.org/10.1080/10720160008400205

Cooper, A., y Griffin-Shelley, E. (2002). The internet:The next sexual revolution. In A. Cooper (Ed.), Sex \& the internet: A guidebook for clinicians (pp. 1-15). Brunner-Routledge.

Cooper, K., Quayle, E., Jonsson, L., y Svedin, C. G. (2016). Adolescents and self-taken sexual images: A review of the literature. Computers in Human Behavior, 55, 706-716. https://doi.org/10.1016/j.chb.2015.10.003

Courtice, E. L., y Shaughnessy, K. (2018). The Partner Context of Sexual Minority Women's and Men's Cybersex Experiences: Implications for the Traditional Sexual Script. Sex Roles, 78(3-4), 272-285. https://doi.org/10.1007/s11199-017-0792-5

Daneback, K., Ševčíková, A., Månsson, S. A., y Ross, M. W. (2013). Outcomes of using the internet for sexual purposes: Fulfillment of sexual desires. Sexual Health, 10, 26-31. https://doi.org/10.1071/SH11023 
Grubbs, J. B., Kraus, S. W., y Perry, S. L. (2019). Self-reported addiction to pornography in a nationally representative sample: The roles of use habits, religiousness, and moral incongruence. Journal of Behavioral Addictions, 8(1), 88-93. https://doi.org/10.1556/2006.7.2018.134

Henry, D. B., Tolan, P. H., y Gorman-Smith, D. (2005). Cluster analysis in family psychology research. Journal of Family Psychology, 19(1), 121-132. https://doi.org/10.1037/0893-3200.19.1.121

O'Sullivan, L. F. (2014). Linking online sexual activities to health outcomes among teens. In E. S. Lefkowitz \& S. A. Vasilenko (Eds.), New directions for child and adolescent development (Vol. 2014, Issue 144, pp. 37-51). Wiley Periodicals. https://doi.org/10.1002/cad

Reid, R. C., y Kafka, M. P. (2014). Controversies About Hypersexual Disorder and the DSM-5. Current Sexual Health Reports, 6(4), 259-264. https://doi.org/10.1007/s11930-014-0031-9

Ross, M. W., Mansson, S. A., y Daneback, K. (2012). Prevalence, severity and correlates of problematic sexual Internet use in Swedish men and women. Archives of Sexual Behavior, 51(2), 459-466. https://doi.org/10.1007/s10508-011-9762-0

Shaughnessy, K., Byers, E. S., y Walsh, L. (2011). Online Sexual Activity Experience of Heterosexual Students: Gender Similarities and Differences. Archives of Sexual Behavior, 40(2), 419-427.

Simon, L. E., Daneback, K., y Ševčíková, A. (2015). The Educational Dimension of Pornography: Adolescents' Use of New Media for Sexual Purposes. In P. Lorentz, D. Smahel, M. Metykova, y M. F. Wright (Eds.), Living in the digital age: self-presentation, networking, playing, and participating in politics (pp. 33-48). Muni Press.

Smith, M. (2013). Youth Viewing Sexually Explicit Material Online: Addressing the Elephant on the Screen. Sexuality Research and Social Policy, 10(1), 62-75. https://doi.org/10.1007/s13178-012-0103-4

Voon, V., Mole, T. B., Banca, P., Porter, L., Morris, L., Mitchell, S., Lapa, T. R., Karr, J., Harrison, N., Potenza, M. N., y Irvine, M. (2014). Neural correlates of sexual cue reactivity in individuals with and without compulsive sexual behaviours. PloS One, 9(7), e102419. https://doi.org/10.1371/journal.pone.0102419 